\title{
High throughput satellites in 5G and MIMO interference limited communications
}

\author{
Ana Pérez-Neria ${ }^{1,2, a}$, Miguel A. Lagunas ${ }^{1,2}$ and Miguel A. Vázquez ${ }^{1}$ \\ ${ }^{1}$ CTTC, Centre Tecnològic de Telecomunicacions de Catalunya, 08860 Castelldefels, Spain \\ ${ }^{2}$ UPC, Universitat Politècnica de Catalunya, 08034 Barcelona, Spain
}

\begin{abstract}
Classically, thermal noise has been the workhorse of satellite communications due to the long distances to be covered between the satellite and the user terminal (UT). Lately, LDPC (Low-Density Parity-Check) codes allow the noise threshold to be set very close to the Shannon limit for the memory-less satellite channel; thus, solving the noise problem that turbo codes were not able to solve. However, recently, the high target rates in next generation 5G wireless terrestrial system are pushing the required spectral efficiency in Satellite Communications; therefore, shifting the SatCom paradigm towards an interference limited one. This paper revisits the 5G scene and the role of next generation satellite communications, with a special focus on high throughput satellites (HTS) together with the future accompanying MIMO interference mitigation techniques.
\end{abstract}

\section{Introduction}

$5 \mathrm{G}$ is conceived as a composition of: i) enhanced mobile broadband; ii) ultra-reliable and low latency communications; iii) massive machine type communications [1]. However and more importantly it is seen as a new infrastructure that will bring a new unique network and service capability to provide an order of magnitude improvement in different key performance indicators. While too much focus has been set so far in urban scenarios, the $5 \mathrm{G}$ ecosystem should continue to adapt their mobile use cases and deployments. Emerging use cases that have been identified are: rural infrastructure, high bandwidth because of many users, but low bandwidth to each one, broadband mobile connectivity. The satellite component can have many things to say in the use cases aiming at helping to bridge the digital divide in parts unreachable by terrestrial, whilst optimizing the value for money to the end-users [2-3]. Ubiquitous coverage, together with resilient overlay communications and disaster relief are the three main assets that satellite can complement terrestrial communications.

Satellite orbits can be classified mainly into three main types: Geostationary (GEO) orbits $(36.000 \mathrm{~km}$ above the equator), Medium earth orbits (from 6000 to $25000 \mathrm{~km}$ altitude) and Low earth orbits $(<1500 \mathrm{~km}$ altitude). Whenever these orbits are combined, hybrid orbits arise. Concerning the services they provide, Fixed Satellite Services (FSS) operate at C band (3-6 GHz), whenever extreme wide coverage and/or resilience to bad weather conditions are needed. This band was the first to be used and currently FSS are moving to Ku band (12-18 $\mathrm{GHz}$ ) and $\mathrm{Ka}$ band (26.5-40 GHz), mainly to provide

\footnotetext{
a Corresponding author: ana.perez@cttc.es
}

backhauling services to terrestrial operators. Mobile Satellite Service (MSS) operate primarily in L band (1-2 $\mathrm{GHz}$ ) and $\mathrm{S}$ band (2-4 GHz). They offer services to both handheld and vehicle mounted terminals as well as some fixed terminals. In the past the air interfaces and networks have been mainly proprietary (e.g. BEGAN for MSS) and recently satellite systems have adopted standards within the DVB-S, ETSI and ITU. Either in FSS or in MSS, the supported services by SatCom are not limited to basic data speeds (with a $5 \mathrm{G}$ target of up to $1 \mathrm{Gbps}$ in average per user for mobile broadband) and voice services, but also for machine type communications, broadcast and narrow band services (up to $100 \mathrm{Kbps}$ per user in machine type communications).

So far, the major business and focus are: FSS-DTH (Direct To Home) for new High Definition (HD), Ultra HD, 3D TV. Another important market is IP trunking to connect parts of the backbone, either for internet or for VoIP, at 310 Mbps. Residential broadband is another important data communication application with 2.20 Mbps for the end user that are evolving towards higher rates. Cellular backhauling for connecting cellular radio towers to the core network are currently working at 2 Mbps and, finally, corporate and public VSAT for connecting a site to the IT network of a private or public organization. These applications are carried out through GEO satellites and, while C-band use keeps flat in forthcoming years, $\mathrm{Ku}$ and $\mathrm{Ka}$ band use increases.

When talking about non-GEO constellations, there are existing LEO constellations that target lower capacity global services. Also, new Mega-constellations are being developed (e.g. OneWeb (640), LeoSat (80-100), SpacEx (4000), Samsung (4600), SSI (80), etc.) and foresee to 
reach by 2020 a Global market with high capacity and low latency services.

Figure 1 sketches a satellite communication system. The Forward Link supports communications from a Gateway (GW) to the Users. The Return Link supports the communications from the Users to the GW. The main features with respect to a terrestrial system are: i) long propagation delay (i.e. $500 \mathrm{~ms}$ from ground station to the satellite and back, known as Round Trip Delay); ii) Doppler effects are relevant for LEO and MEO constellations (i.e. $200 \mathrm{KHz}$ for high speed UEs). Whenever a hybrid terrestrial/satellite system is the target, new interfaces should accommodate these features to the baseline terrestrial system. In fact, more than a mere relay, the satellite should be an integral part of the network [4].

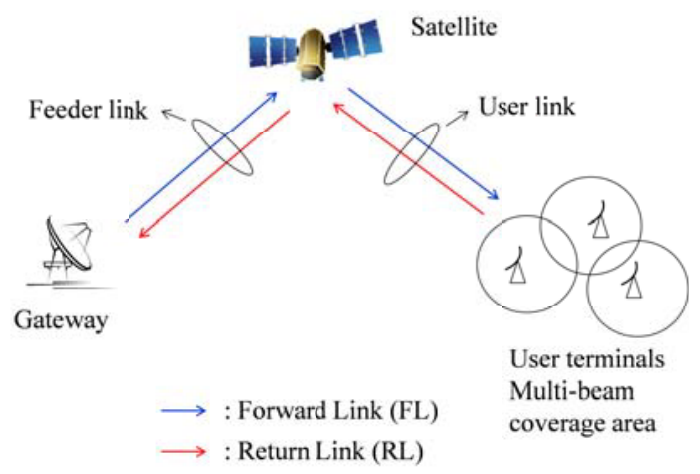

Figure 1. Satellite system.

A possible classification of the satellites focuses on their beam topology, namely: number of beams, radius of each beam and flexibility when configuring the beams. High Throughput Satellites (HTS) are considered the next generation of GEO satellites [5-6], which split the service area into a multi-spot service area to exploit a high order of frequency reuse and use the ensuing satellite antenna gain to deliver more capacity for given satellite resource. HTS systems can be classified by the capacity they provide. The 1st generation offered an aggregate capacity of up to a few 10's of Gbit/s. The 2nd generation, led by Ka-Satellites, offers approx. 100Gbit/s. To satisfy the technology agnostic consumer, the system must deliver services comparable to fiber-to-the-home and 3rd generation HTS systems will go a step further and offer a few hundred Gbit/s. Because of its broadband nature and potential to lower the cost per bit, HTS is a potential wild card to help in attaining many of the 5G network requirements.

\section{High Throughput Satellites}

The most valuable asset in any given orbital location is spectrum. High throughput satellites employ multiple spot beams to enable "frequency re-use". This is achieved typically with 4 color re-use (two frequency slots and two polarizations) and high gain spot beams. Whilst GEOHTS multi-spot/beam satellite configurations can be applied to $\mathrm{Ku}$ and $\mathrm{C}$ frequency bands, such systems are particularly applicable to the Ka frequency band, which allows: better use of the reflector aperture size, very small beam sizes of increased gain with high roll-off to neighboring beams and benefit from smaller and lighter feed technologies that enable accommodation of tightly packed beams. Currently they have around 100 beams and provide an aggregate rate of $100 \mathrm{Gbps}$ at $\mathrm{Ka}$ band. Higher gains allow for improvements to spectrum efficiency via higher order coding and modulation (16APSK vs 8SPK for example). Future GEO-HTS systems will use exclusive and shared Ka-bands [4], flexible power distribution, dynamic beamforming, beam hopping, etc...

In HTS, user service areas are typically located over a large region and therefore a large number of cells are required. Frequency Reuse (FR) is applied such that the same frequency on a given polarization is reused several times. This is achieved through separation of the cells to control the intra-system interference. This is achieved typically with 4 color re-use (two frequency slots and two polarizations) and high gain spot beams. Figure 2 shows an example of HTS Europe coverage. For HTS systems, in order to provide good performance, the antenna should have high gains with steep roll-offs and low side lobes that result in good C/I. The figure plots only the footprints of each beam. Active antennae's could help in the efficient use of spacecraft power.

The feeder link (see Figure 1) is considered ideal in terms of Signal to Noise Ratio (SNR) and it is only bandwidth limited. The user link is limited by the additive noise, in terms of SNR, and by the generated interference, in terms of Signal to Interference Ratio (SIR). Therefore its link budget determines the performance. In each beam/color, the downlink user signal is typically a time multiplex signal e.g. DVB-S2 such that at the satellite level, this can be considered as a single continuous signal from fixed angular offsets. Assuming infinite lattice of 4 color reuse, Figure 3 shows that each cell has 6 first order neighbors of the same color. With the signal structure this implies that interference for a given location in the wanted center cell is static. The performance is a function of the location of the terminal in the cell and is further degraded by the repeater C/I effects and the inter-spot interference.

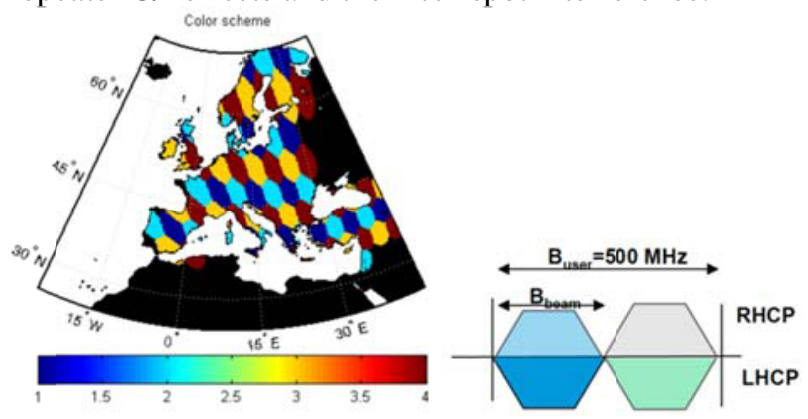

Figure 2. HTS applied to a European coverage with $500 \mathrm{MHz}$ user bandwidth divided in 4 colours. Each color is up to $250 \mathrm{MHz}$ on one polarization. 


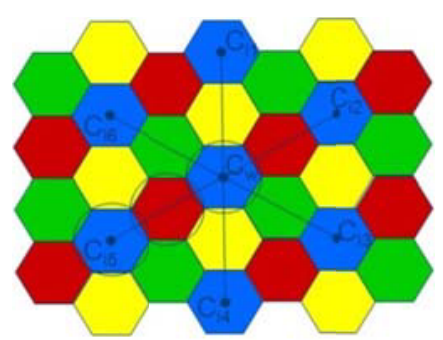

Figure 3. First order cluster of interfering cells with $\mathrm{FR}=4$.

In order to increase capacity, next HTS systems are considering more aggressive frequency reuse (i.e. the target is reuse 1 or 2); thus, increasing interference. In other words, in order to make full use of diversity schemes, orthogonality is not imposed anymore. This fact urges the need for advanced interference management techniques that enable the satellite system to work in an interference limited scenario, rather than in a noise limited one. New LDPC codes allow to approach the theoretical Shannon limit (DVB-S2, S2X, RCS2, ..), but are not able to properly handle interference on its own. Before getting into the study of PHY layer alternatives to manage interference, we devote the rest of this section to revisit one of the major features of HTS, which is its ability to have flexible payloads. This aspect is considered key in order to have higher throughput per satellite. Tremendous evolution in HTS payload capabilities is foreseen: in 2003 typical systems offered 2-4 Gbps, while in 2016, 1000 Gbps are proposed.

In order to lower the cost per bps, the key aspect in multi beam HTS is its flexibility to control the peak rate per spot beam while guaranteeing an average rate within the service area and minimum rate at the edges. In order words, HTS takes advantage of hot and cold spots. The capacity depends on many system choices: antenna size, technology, network dimensioning ... The traffic profile is highly time and region dependent; thus, traffic peaks are not geographically synchronous. For instance, while with one wide footprint a peak rate of $54 \mathrm{Mbps}$ and a maximum of 5475 users can be reached. With 8 beams covering the same area, the attained sum peak rate can be 123 Mbps. Traffic peaks also present a daily repetition. Namely, the flexibility introduced by HTS helps counteract the regional peak demands per beam. Concerning the cost, a possible approach here is to leverage on the traffic statistics for reducing the DC Power consumption and to reflect this on the price to be paid by the user. We note that broadcast bent-pipe payload architectures do not have this need or problem.

Payload flexibility techniques are relatively well-used in the current generation of HTS systems in order to: i) reconfigure the bandwidth allocation per beam (i.e. thanks to digital or analogue on-board switching and filtering); ii) reconfigure the power allocation with the user of Flex amplifiers. In future systems, beam hopping will be possible in order to jointly allocate time, power and bandwidth to each beam. Also, flexible beam coverage is foreseen.

Additional payload flexibility requires On Board Processing (OBP) in order to regenerate the signal and to carry out beamforming at the satellite. The goal is to have further SNR and SIR gains. However, OBP has a cost and a trade-off with other system and business case aspects needs to be done to justify that cost factor.

Beam hopping is one of the most popular actual alternatives in order to confer flexibility to the system. It means: time sharing according to a beam hopping pattern. In other words, at any given time, only a subset of beams is illuminated. The illumination time of any beam can be changed according to the traffic request of that beam. In addition, power and bandwidth can be flexibly allocated. The constraint is that the total illumination time of the set of beams served by a High Power Amplifier (HPA) has to stay constant. Therefore, the flexibility of throughput is within the set of beams illuminated by the same HPA. There is a total number of time slots that that can be assigned to the beams connected by the same switch. The minimum slot duration depends on the minimum switching time and defines the minimum capacity granularity of the network. By assigning different time slots to different beams the capacity per beam is adjusted. Figure 4 depicts an example of time and beam assignment for beam hopping within 6 time slots.

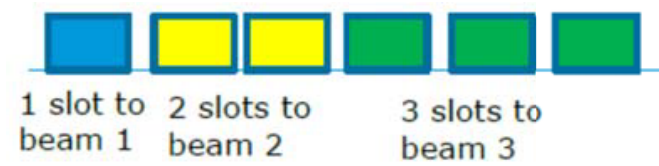

Figure 4. Time and beam assignnnent for beam hopping within 6 time slots.

The capacity values of a beam hopping and a frequency division multiplex are the same if the number of beams, HPAs and user bandwidth, are identical. However, such two systems would correspond to a different overall payload mass, resulting heavier the frequency division multiplex.

When selecting appropriate technologies and architectures for flexible HTS payloads the network architecture and its resulting impacts to geographical connectivity need to be considered. Currently, new Mesh or Open Architectures are being researched by payload manufacturers in order to get advantages over the classical Star or Closed Architectures. In mesh or Open Architectures:

- Satellite Capacity (MHz) and Uplink access are provided.

- The User is responsible for network definition and operations.

- $\quad$ Payload / Repeater need to provide geographical connectivity implying a mesh connectivity matrix

- Digital payload or beam hopping adds value to manage complex connectivity.

In contrast, when using the satellite within a star architecture conception:

- The operator defines the network and sells end to end service based on Mbps

- The access to the network is through operator gateways 
- Payload / Repeater is typically a star network with well-defined gateway to user beam paths

Therefore, connectivity matrices between any uplink channel and any downlink beam, as shown in Figure 5, can become major payload design drives as the number of beams in an HTS system increases.

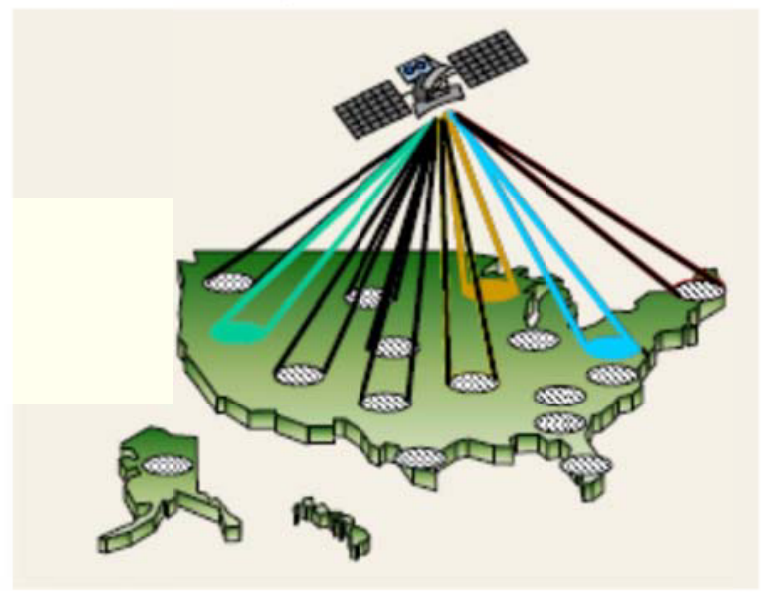

Figure 5. The satellite in a Mesh architecture

Digital processing capabilities at the satellite are desirable to have full flexibility. Topics of current research are: channelization and connectivity, digital on board beamforming, low consumption DAC and ADC, optimization of input gain and dynamic range (i.e. tradeoff between noise figure and dynamic range), currently there is a total signal power limit of $-8 \mathrm{dBm} / 500 \mathrm{MHz}$ and gain must be sufficient to map small carriers above port quantization noise floor. Finally, the radio frequency payload performance to manage has to be analyzed as there are multiple additional potential degradations to payload Es/No to manage, such as: Phase Noise, Frequency Stability impacts for narrow band channelization, Gain frequency response \& Group Delay, Clock Jitter, Alias Band Folding. It is important to understand the relative impact of these in order to optimize the balance between payload performance and equipment cost.

In short, HTS and flexible payloads open a variety of topics to explore. Section 4 summarizes those that are related to signal processing. Before that, next Section 3 focuses on those signal processing techniques that are being researched in order to manage interference. HTS stresses the spectrum usage in order to reduce costs; thus, changing the noise limited paradigm of satellite communications into interference limited.

\section{Interference Mitigation Techniques: MIMO processing}

HTS and multi-beam satellites in general will change the TV distribution business in coming years, among others. One aspect of HTS that has been mentioned in the previous section is the introduction of greater amounts of radio frequency interference, primarily through frequency reuse with multiple spot beams on the same frequency [7]. As a result, beam to beam interference becomes the limiting factor. This fact translates the paradigm of satellite communications as noise limited ones into interference-limited communications; thus, attracting more research efforts towards these systems.

There are various means by which interference can be managed. In this section we focus on those situations where the satellite communication can be modelled as a Multiple Input Multiple Output (MIMO) system [8-12]. Thus, multiple signals coexist geographically in time and in frequency. The goal is first to set up the capacity bounds that are benchmarking the system. Next, an overview of the signal processing techniques that solve the interference problem come. The radio resource management that should be considered to optimize the usage of code, power, bandwidth and beams in a dynamic way is out of the scope of this paper and interesting techniques can be found in [13]. This section compiles these different aspects with the aim to help in future research.

\subsection{System model}

Let us assume that at a given time instant and frequency bean, $K$ signals, $s_{i}, i=1 \ldots K$, are to be simultaneously transmitted through different physical channels. The $K$ received signals are $x_{i}, i=1 \ldots K$. The base-band transmission can be modelled as

$$
\boldsymbol{x}=\boldsymbol{H B S}+\boldsymbol{w}
$$

where $\boldsymbol{x}$ and $\boldsymbol{s}$ are complex vectors that contain the received and transmitted signals respectively. Vector $\boldsymbol{w}$ contains thermal noise samples of Gaussian distribution with zero mean and variance equal to $N_{o}$ Watts. The elements of matrix $\boldsymbol{H}$ are $h_{i j}, i, j=1 \ldots K$, and model de equivalent channel gains that affect the transmission of symbol $s_{j}$ when it is received by therminal $i$. These gains are random variables, whose statistics depend on the specific situation that they are modelling. If matrix $\boldsymbol{H}$ is not diagonal, each terminal $i$ will not only receive its intended signal $s_{i}$, but also interference from the others. The role of matrix $\boldsymbol{B}$ is to counteract this interference and its design is the focus of study of MIMO precoding techniques.

Depending on the system architecture and constraints that (1) is modelling, additional MIMO processing has to be carried out at reception on signal $\boldsymbol{x}$. Finally, if (1) faces a multiuser system, scheduling techniques should be designed in order to select the most suitable set of $K$ users out of a bigger set of $N$ possible users. Model (1) is a time and frequency snapshot, therefore it only contains a spatial model as bandwidth or time allocations are out of the scope of the present review.

First, it is of interest to analyze when model (1) is suitable for a satellite transmission. In this respect, there were initial studies promoted by the European Space Agency with this purpose [8-9]. The conclusion is that direct application of MIMO to satellite in a single-user scenario is rather limited due to : i) strong LOS component and ii) absence of rich scattering environment. Therefore, the resulting MIMO channel $\boldsymbol{H}$ is rank deficient and the optimal design is to transmit a single stream and use beamforming in order to point to the desired user. In other words, there is not MIMO 
processing as such. MIMO processing is needed only in situations where there is significant movement and multipath appears. One example is GEO maritime transmission [14].

Still, if either the Multi Beam dimension, or the Multi Satellite dimension, or the Multiple Gateway dimension, or even the Hybrid satellite-Terrestrial networks are considered, MIMO processing is needed. All these situations use to be multiuser communications instead of single user ones.

Before getting into the MIMO processing techniques, next some capacity bounds from (1) are considered [15].

\subsection{MIMO Capacity}

Whenever model (1) can be applied, if the spatial degrees of freedom are properly used, there is additional capacity per $\mathrm{Hz}$ and second. In a scenario with $N$ total users and $K$ possible simultaneous transmissions (i.e. $K$ transmitters and $K$ receivers), if the transmitter has all the channel state information (CSIT) and so the receiver does (CSIR), the maximum attained spectral efficiency is

$$
\begin{aligned}
& C_{S N R \rightarrow \infty}=\min (N, K) \log (S N R) \\
& C_{N \rightarrow \infty}=K \log \log (N K)
\end{aligned}
$$

Whereas, if only CSIR is availble, the maximum spectral efficiency is

$$
\begin{aligned}
& C_{S N R \rightarrow \infty}=N \log (S N R) \\
& C_{N \rightarrow \infty}=0
\end{aligned}
$$

In expressions (3) and (5) the total number of users tends to infinite in order to highlight the so-called multiuser diversity gain: whenever the set of users that require transmission is high, there is an inherent system gain, even though the system can only set transmission with one user at a time. Note that there is no multiuser capacity gain if only CSIR is available (i.e. (5) is zero).

Next section gets into the signal processing techniques that are required to approach the mentioned bounds. As the goal is to study the multiple beam scenarios, which is a multiuser scenario, next section focuses on this multiple user case.

\subsection{MIMO multiuser in multi-beam satellite}

As it has been previously commented, in order to increase capacity, next HTS systems are considering more aggressive frequency reuse (i.e. the target is reuse 1 or 2); thus, increasing interference.

\subsubsection{On ground processing for MIMO precoding}

Let us consider frequency reuse 1 with the simplest satellite system configuration when only one gateway is serving $\mathrm{K}$ users (as Figure 1 depicts). With frequency reuse 1 , the GW aims to serve simultaneously $\mathrm{K}$ users, all of them sharing the same $500 \mathrm{MHz}$ band, and different information is to be conveyed to each user (so-called unicast transmission). The specific model for $\boldsymbol{H}$ can be found in [9]. In this situation MIMO processing can be done in the forward link at the transmitter in order to diminish the interference with a suitable design of matrix $\boldsymbol{B}$ in (1). If full CSIT is available, linear processing techniques such as Zero-Forcing or its regularized version: the MMSE (Minimum Mean Square Error) precoding can be implemented [16]

$$
\begin{aligned}
& \boldsymbol{B}_{Z F}=G_{Z F} \boldsymbol{H}^{H}\left(\boldsymbol{H} \boldsymbol{H}^{H}\right)^{-1} \\
& \boldsymbol{B}_{M M S E}=G_{M M S E} \boldsymbol{H}^{H}\left(\alpha \boldsymbol{I}+\boldsymbol{H} \boldsymbol{H}^{H}\right)^{-1}
\end{aligned}
$$

If more complex processing is allowed, non-linear precoding alternatives can be applied in order to attain a performance close to the bounds in (2) and (3). If only partial channel state information is available at the transmitter, opportunistic beamforming is another very interesting alternative [17]. Figure 6 depicts the total average throughput that is obtained with these precoding techniques and compares them with the frequency reuse 4 scenario that serves as a reference. Note that precoding improves performance both in terms of throughput and availability, especially with the linear MMSE precoder. An important aspect is that in this scenario the satellite behaves just as a transparent payload; thus no on board processing is needed. All the pre-processing is carried out on ground, at the gateway. In [16] the reader can find further studies on how to cope with: imperfect CSIT, fairness, availability and bigger number of feeds at the satellite than $K$ users to simultaneously transmit. Current challenges are to match these designs with the DVB S2x frame, where all simultaneous users should use the same spatial precoder instead of the $K$ different ones that are encompassed in equations (6) and (7). In other words, the design should be multicast instead of unicast and the same precoder must fulfill different Quality of Service at the same time [18-19].

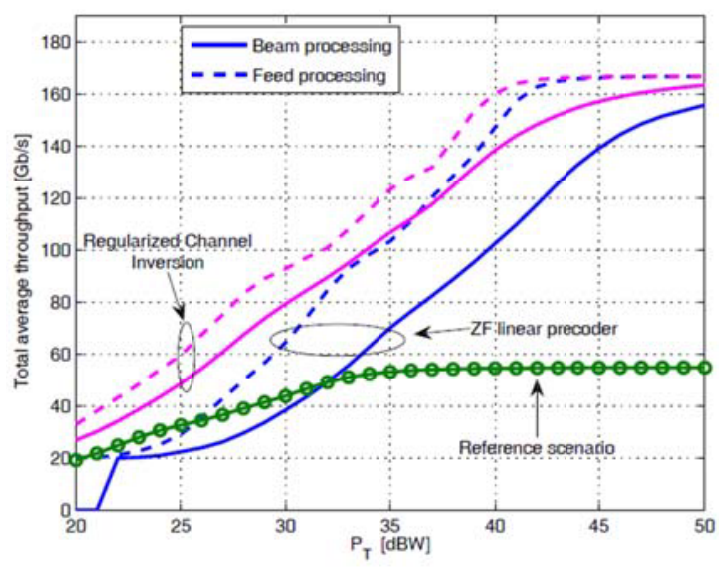

Figure 6. GEO satellite for FSS. Total average throughput in a $500 \mathrm{MHz}$ system with frequency reuse 1 and MIMO precoding on ground. Results are compared with a frequency reuse 4 reference system.

The more users can be served in the user link, the higher the capacity should be in the feeder link. This fact is currently motivating research in two directions: go up to $\mathrm{Q} / \mathrm{V}$ and optical bands in the feeder link or, use multiple gateways to give service to the same number of beams. This later topic triggers further research on MIMO processing because a proper cooperation and channel information exchange has to be designed to make the system practicable [20]. 


\subsubsection{Multiuser decoding at the user terminal}

In the previous section, the transmitter was in charge of pre-cancelling the interference; therefore, all the processing complexity remained at the gateway. Another alternative is to increase the complexity of the user terminal, which will carry out multiuser decoding in order to eliminate interference. In other words, whenever the user terminal receives its desired signal contaminated by interferences that operate in the same frequency band one alternative is to demodulate them so that they can be properly subtracted from the incoming signal (i.e. the socalled Sequential Interference Canceller or SIC). In this way, the desired stream can be recovered finally clean from interference. The stronger the interference, the better it can be cancelled with this strategy. Next we consider single antenna user terminals (UTs), techniques with array processing at the UT are also possible at the cost of increasing complexity [21].

Let us consider, for instance, a frequency reuse 2, such as in Figure 7, which shows the footprints of a multi-beam satellite over European coverage in this case.
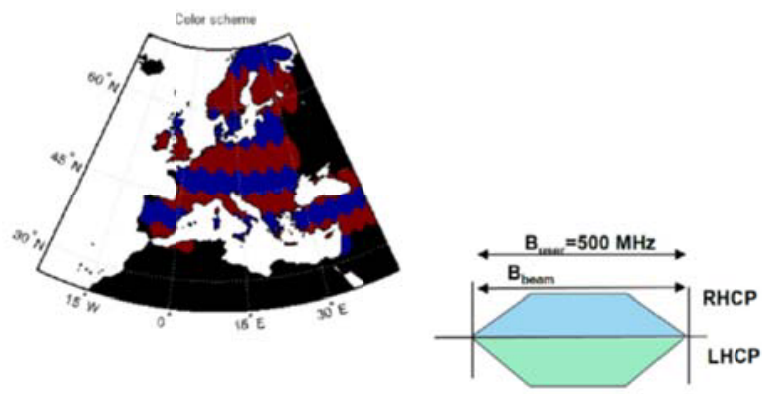

Figure 7. HTS applied to a European coverage with $500 \mathrm{MHz}$ user bandwidth divided in 2 colours. Each color is up to $500 \mathrm{MHz}$ on one polarization.

In this situation multiple beams serve simultaneously overlapped areas. Depending on the position of the user within each beam coverage area, the level of interference will vary. Figure 8 plots the distribution of possible $\frac{C}{I_{1}}$.

Table 1. Interference levels up to the $6^{\text {th }}$ tear for $F R=2,4$.

\begin{tabular}{|c|c|c|c|c|c|c|}
\hline & Reference & Int. 1 & Int. 2 & Int. 3 & Int. 4 & Int. 5 \\
\hline $\mathrm{FR}=2$ & $P$ & $\frac{p}{l_{1}}=6 \mathrm{~dB}$ & $\frac{F}{l_{2}}=27 \mathrm{~dB}$ & $\frac{p}{h_{1}}=26 \mathrm{~dB}$ & $\frac{F}{L}=27 \mathrm{~dB}$ & $\frac{P}{l_{\mathrm{s}}}=30 \mathrm{~dB}$ \\
\hline$F R=4$ & $P$ & $\frac{T}{L}=22 \mathrm{~dB}$ & $\frac{\beta}{t_{1}}=22 \mathrm{~dB}$ & $\frac{\eta}{h_{1}}=22 \mathrm{~dB}$ & $\frac{7}{L}=22 \mathrm{~dB}$ & $\frac{p}{l_{k}}=22 \mathrm{~dB}$ \\
\hline
\end{tabular}

Users located in the intersection of beams can benefit from multi-user detection together with scheduling techniques to boost capacity.

In the implementation of the multiuser decoding the modulation and coding of the desired signal and that of the interference matters in practice. Figure 9 and 10 show the different performance that can be obtained [2224]. Therefore, multiuser detection has to be carried out together with joint adaptive modulation. In order to allow a practical user scheduler, one possible strategy is to to use both signals (transmitted from two co-channel beams) to carry useful information for the considered user. Then, both transponders (beam 1 and beam 2) are used to serve one user at a time. Figure 11 shows that with this joint detection and scheduling strategy capacity increases with respect to the frequency reuse 4 baseline scenario.

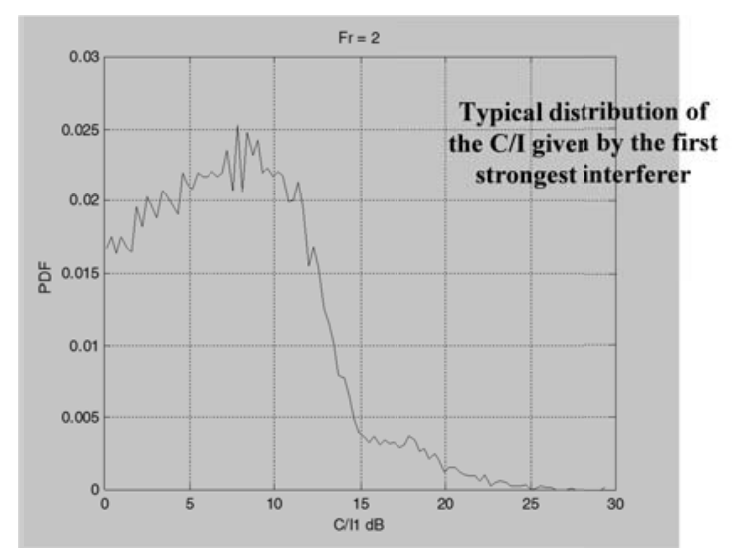

Figure 8. Probability Density Function of the Carrier to main Interference Ratio $\left(\frac{C}{I_{1}}\right)$ with frequency reuse 2 .

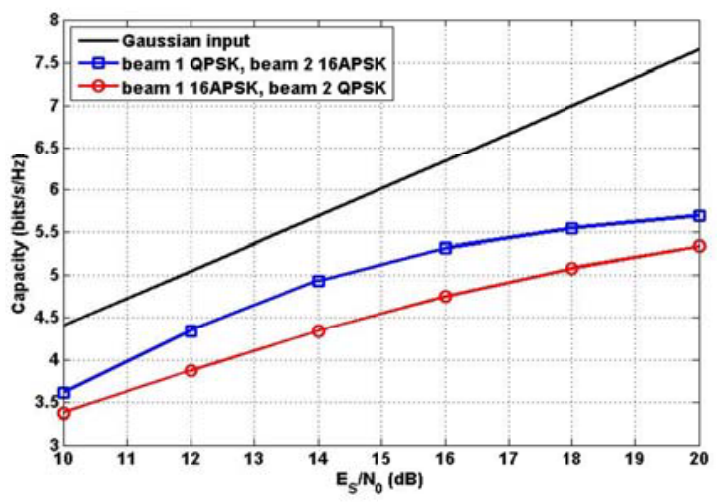

Figure 9. Capacity for user in beam 1 when beam 2 reuses the frequency and acts as an interference with $\left(\frac{C}{I_{1}}\right)=0 \mathrm{~dB}$.

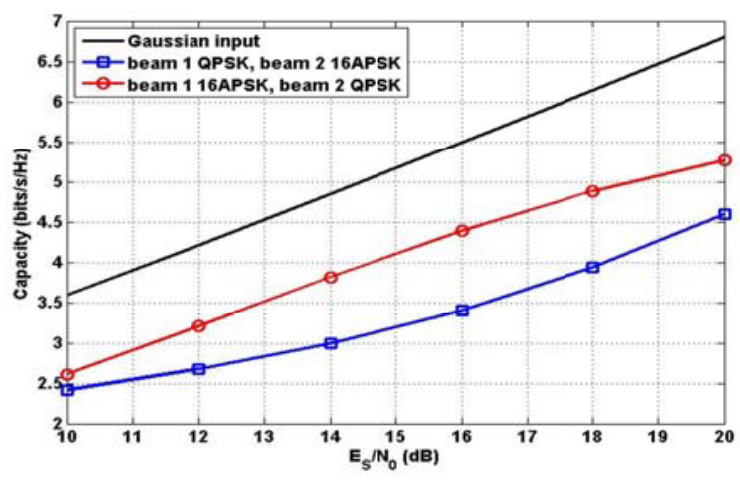

Figure 10. Capacity for user in beam 1 when beam 2 reuses the frequency and acts as an interference with $\left(\frac{C}{I_{1}}\right)=10 \mathrm{~dB}$.

\subsubsection{Joint transmit and recieive MIMO processing}

In the forward link of a multi-beam satellite networks we have differentiated so far between techniques employed at the transmitter (gateway, GW) and techniques employed at the receiver (UT). If the goal is to attain rate performance close to the capacity bounds, the system should take advantage of both and carry out a joint precoding/detection design: i) the scheduler considers the possibility of superposition coding or joint ACM among the different beams; ii) the precoder uses power control 
and the interference rejection capabilities of the receivers. This is a matter of future research.

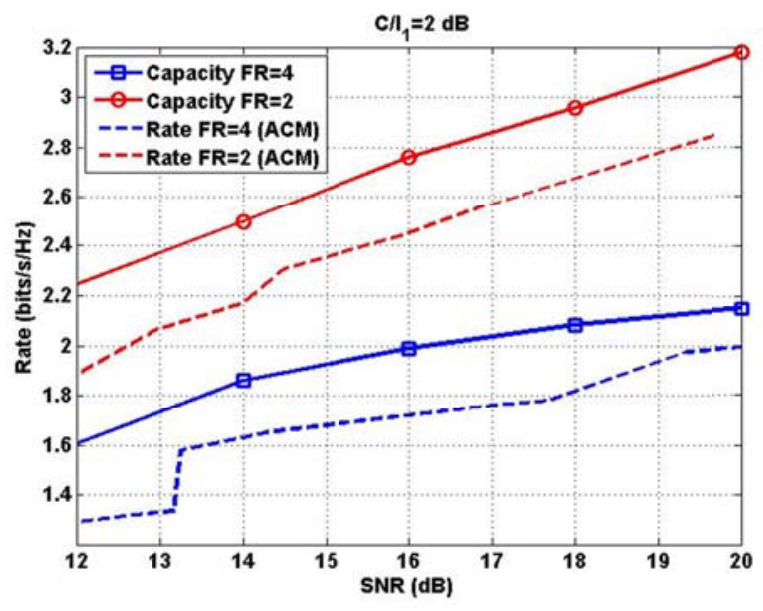

Figure 11. Aggregate capacity and rate for $F R=2$ with joint multiuser decoding and scheduling. In the $\mathrm{FR}=4$ scenario the detection is single user.

\section{Signal Processing Challenges}

Next Generation wireless terrestrial networks will not provide ubiquitous communications. In contrast, satellite networks provide coverage, disaster relief and resilience and therefore, both networks are complementary. The novelty and challenges are [25] : 1) New network architectures and constellations, LEO, MEO; 2) Aeronautical and Professional Link Designs for Satellite Systems at Extremely High Frequency Bands; 3) Seamless services over hybrid terrestrial-satellite networks; 4) Optical free-space communications, 5) Onboard signal processing and 6) Multiple Gateway architectures.

In each of the challenges that have been identified novel tailored solutions for the satellite segment have to be studied. If we focus now on the physical layer aspects and the associated signal processing problems,

- concerning the integration of terrestrial and satellite systems in future 5G networks, there are different aspects to be considered from a waveform perspective because frequency assignments range from $300 \mathrm{MHz}$ up to 100 $\mathrm{GHz}$ and both, Single- and multi-carrier solutions have to be studied, together with

- Licensed/shared/unlicensed spectrum access.

- As in the wireless arena, not only orthogonal but also non-orthogonal multiple access can be useful to increase the spectral efficiency; thus, creating the need for interference management techniques.

- There are also several impairments in a satellite channel to be dealt with: phase noise, nonlinearities, on-board filtering.

We talk about tailored solutions because it has to be studied: i) how do the proposed air interface(s) performs in the presence of typical satellite channel impairments and ii) how do the proposed countermeasures devised for terrestrial channel impairment fit satellite architectures. In this latter issue, note that satellite communications present, not only a different channel, when compared to the wireless terrestrial one, but also, as it has been commented along the text, there are some specificities such as:

- $\quad$ long round trip delay (that can be overcome by addressing the proper service and cache management);

- $\quad$ satellite high power amplifiers (HPAs) effects on the signal;

- Limited on-board power, mass and accommodation yields to limitation in terms of HPAs, when dealing with high-frequency re-use and precoding;

- frame structure that encompasses many users under the same channel code in order to properly combat noise (that forces many times a multicast configuration rather than a unicast one);

- GW scheduler strategies modification to be in line with precoding techniques;

- geographical and time traffic variations that are different from the terrestrial only communications (e.g. a satellite can serve geographical areas that are in different time-slots and with complementary traffic needs);

- trade-offs between capacity, availability and fairness requirements that become more relevant than in the terrestrial arena;

- channel variations that depend not only on the user terminal, but also on the satellite orbit and can range from very slow ones for GEO satellites and FSS to high Doppler with LEO satellites;

- synchronization and time recovery issues different from the wireless terrestrial segment for channel estimation and interference cancellation;

- on-ground and on-board complexity considerations.

Finally, and in addition to the mentioned aspect, if TeraHertz communications are targeted, Very HTS are around the corner and will bring new situations to be solved in order to properly work in $\mathrm{Q} / \mathrm{V}$ band $(40 / 50$ $\mathrm{GHz})$, W band $(70 / 80 \mathrm{GHz})$ and optical bands, with photonics optimized payload mass [26].

\section{Acknowledgements}

This work has been developed within the network of Experts SATNEX IV (http://www.satnex4.org/) that is supported by the European Space Agency. This work has also received funding by the Spanish Ministry of Economy and Competitiveness (Ministerio de Economia y Competitividad) under project TEC2014-59255-C3-1-R (ELISA); and from the Catalan Government (2014SGR1567).

\section{References}

1. 5GPPP Association, 5G Vision Brochure, www.5gppp.eu, (2015)

2. NetWorld2020's-SatCom WG, The role of satellites in 5G, http://www.networld2020.eu (2014) 
3. NGMN Alliance, NGMN White Paper, www.ngmn.org/ (2015)

4. www.sansa-h2020.eu/

5. Y. Vasavada, R. Gopal, C. Ravishankar, G. Zakaria and N. BenAmmar, Architectures for next generation high throughput satellite systems, International Journal of Satellite Communications and Networking (2016)

6. P. M. French, High Throughput Satellites (HTS) are pushing open the satellite market door, guest column Near Earth LLC. (2012).

7. T. Glynn et al, Multi-beam satellite communications system, US patent 20040166801 A1 (2004)

8. G. Gallinaro, G. Caire, M. Debbah, L. Cottatellucci, R. Muller, R. Rinaldo, Perspectives of adopting interference mitigation techniques in the context of broadband multimedia satellite systems, in Proc. 23rd AIAA Int. Commun. Satell. Syst. Conf., ICSSC (2005).

9. N. Zorba, M. Realp, A. Perez-Neira, An improved partial CSIT random beamforming for multibeam satellite systems, in Proc. 2008 10th International Workshop on Signal Processing for Space Communications (2008).

10. A. Perez-Neira, C. Ibars, A, del Coso, M. Caus, MIMO channel modeling and transmission techniques for multi-satellite and hybrid satelliteterrestrial mobile networks, Physical Communication 4 (2011) 127-139, Elsevier (www.elsevier.com/locate/phycom)

11. K. P. Liolis, J. Gómez-Vilardebó, E. Casini, A. Pérez-Neira, Statistical Modeling of MIMO Land Mobile Satellite Channels, IEEE Trans. on Comm., vol. 58, issue 11 (2010)

12. D. Pantelis et al., MIMO over Satellite: A Review, IEEE Communications Surveys \& Tutorials, Volume:13, Issue: 1, (2011)

13. Digital Satellite Communications, ed. By G. Corazza, Springer (2007)

14. P. Henarejos, A. I. Pérez Neira, Dual Polarized Modulation and Reception for Next Generation Mobile Satellite Communications, IEEE Transactions on Communications (Volume:63 , Issue: 10 ) (2015)

15. M.A. Lagunas, A. Pérez-Neira, MIMO processing, class notes at http://www.cttc.es/researchdevelopment/training/graduate-and-undergraduatelectures/

16. J. Arnau, B. Devillers, C. Mosquera, A. Pérez-Neira, Performance study of multiuser interference mitigation schemes for hybrid broadband multibeam satellite architectures, EURASIP Journal on Wireless Communications and Networking (2012)

17. N. Zorba Barah, M. Realp Campalans, A. PerezNeira, Beamforming technique for broadband satellite communications, US8310980 B2 patent (2012)

18. N. Zorba, A. Pérez-Neira, Performance Evaluation of MIMO Multiuser Opportunistic Schemes under QoS requirements, in 4G Mobile \& Wireless
Communications Technologies, River Publishers, Aalborg, Denmark (2008) ISBN 978-8792329028.

19. D. Christopoulos, S. Chatzinotas, G. Taricco, M. Á. Vázquez, A. I. Pérez-Neira, P. Arapoglou, A. Ginesi, Multibeam joint precoding: Frame-based design, Chapter in Cooperative and Cognitive Satellite Systems", edited by Symeon Chatzinotas, Bjorn Ottersten, Riccardo De Gaudenzi, published by Elsevier. 11 (2015). ISBN: 978-0-12-799948-7.

20. V.Joroughi, M. Á. Vázquez, A. I. Pérez-Neira, Multiple Gateway Precoding with Per Feed Power Constraints for Multibeam Satellite Systems, in Proceedings of European Wireless 2014 (EW'14), 14-16 May 2014, Barcelona (Spain).

21. J. Grotz, Interference Mitigation and Synchronization for Satellite Communications, $\mathrm{PhD}$ Thesis (2008)

22. M. Caus, A. I. Pérez-Neira, M. Angelone, A. Ginesi, An innovative interference mitigation approach for high throughput satellite systems, Proc. IEEE International Workshop on Signal Processing Advances in Wireless Communications, SPAWC (2015).

23. G. Cocco, M. Angelone, A. I. Perez-Neira, Cochannel interference cancellation at the user terminal in multibeam satellite systems, in Proc. 7th Advanced Satellite Multimedia Systems Conference and the 13th Signal Processing for Space Communications Workshop (ASMS/SPSC), (2014), pp. 43-50.

24. M. Angelone, A. Ginesi, M. Caus, A. I. Perez-Neira, J. Ebert, System Performance of an advanced multiuser detection technique for high throughput satellite systems, Proc. 21st Ka band and broadband communication conference (2015).

25. EURASIP, Position Paper on Signal Processing Challenges for Communications beyond 5G, Joint Expert Group and 5G Vision Working Group Workshop, Bologna 16 April (2016)

26. O. Vidal et al., Next Generation High Throughput Satellite System based on Optical Feeder Links, Proceedings of the $\mathrm{Ka}$ and Broadband Communications, Navigation and Earth Observation Conference (2015) 